Cross-sectional associations between wake-time movement behaviour compositions and indicators of mental health among preschool children

Denver M. Y. Brown,,${ }^{1,2}$ Matthew Y. Kwan, ${ }^{2,3}$ Sara King-Dowling ${ }^{4} \&$ John Cairney ${ }^{5}$

${ }^{1}$ Department of Psychology, University of Texas at San Antonio, San Antonio, Texas, USA; denver.brown@utsa.edu;

${ }^{2}$ Infant, Child and Youth Health (INCH) Lab, Department of Family Medicine, McMaster University, Hamilton, Ontario, Canada;

${ }^{3}$ Department of Child and Youth Studies, Brock University, St. Catherines, Canada, mkwan@brocku.ca;

${ }^{4}$ Division of Oncology, The Children's Hospital of Philadelphia, Philadelphia, PA, USA, kingdowlis@chop.edu;

${ }^{5}$ School of Human Movement and Nutrition Sciences, University of Queensland, Brisbane, Queensland, Australia, j.cairney@uq.edu.au

Corresponding author: Denver Brown

All authors have agreed that this manuscript is submitted to SportRxiv in its current form.

August 11, 2021

Manuscript type: Original research

Please cite this paper as: Brown, D. M. Y., Kwan, M. Y., King-Dowling, S. \& Cairney, J. (2021). Cross-sectional associations between wake-time movement behaviour compositions and indicators of mental health among preschool children. Pre-print available from SportRXiv.

Keywords: physical activity, sedentary behaviour, daily time use, mental ill-being, Developmental Coordination Disorder 
MOVEMENT COMPOSITIONS AND MENTAL HEALTH

\begin{abstract}
Movement behaviours have been found to be important correlates of health for children and may be particularly important for children with Developmental Coordination Disorder (DCD) who often experience greater mental health problems. To date, however, little research has investigated the daily movement composition of preschool children with Developmental Coordination Disorder (DCD) and/or its association with mental health. The purpose of the current study was to: 1) examine whether differences in movement compositions (i.e., sedentary time, light physical activity, moderate-to-vigorous activity) exist between typically developing (TD) preschool-age children and those at risk for DCD (rDCD); and 2) investigate associations between movement compositions and mental health indicators. This cross-sectional study used the baseline cohort data from the Coordination and Activity Tracking in CHildren (CATCH) study. A total of 589 preschool-age children $\left(M_{\text {age }}=4.94 \pm 0.59\right.$ years; $57.4 \%$ boys $)$ were included in this analysis, of which 288 were classified as rDCD. Wake time movement behaviours were measured using accelerometers and parents completed the Child Behavior Checklist to assess their child's mental health (i.e., internalizing and externalizing problems). Compositional data analysis techniques were used. After adjusting for potential confounders, the results demonstrated similar movement compositions between TD and rDCD children. Among the full sample, findings revealed a significant association between sedentary time and externalizing problems, however, each of the other associations did not reach statistical significance. These results are consistent with emerging evidence demonstrating similar patterns of physical activity and sedentary time among TD children and those classified as rDCD during the preschool years. Although movement behaviours explained little variance in mental health during this period, future research should investigate when movement compositions diverge, and
\end{abstract}


MOVEMENT COMPOSITIONS AND MENTAL HEALTH

how these changes may impact the mental health of TD children and those classified as rDCD

later in childhood. 


\section{MOVEMENT COMPOSITIONS AND MENTAL HEALTH}

Cross-sectional associations between wake-time movement behaviour compositions and indicators of mental health among preschool children

An abundant body of literature has demonstrated the positive impact of moderate-tovigorous physical activity (MVPA) for several developmental and health outcomes in early childhood (Carson, Lee, et al., 2017). However, much of this work has failed to take into account that other movement behaviours - such as sleep, sedentary time and light physical activity (LPA) - also play an important role (Feng et al., 2021). This constrained approach has sparked a push for the adoption and application of the 24-hour movement paradigm to better understand how interrelationships among movement behaviours across the whole day affect healthy development during the early years (Tremblay, 2020; Tremblay et al., 2017). Given that unhealthy patterns of movement behaviours established in the early years could lead to the onset of adverse health outcomes at a young age and potentially have downstream negative consequences across the life course, it is imperative that we improve our current understanding of these relationships so that we can design effective interventions moving forward.

Certain populations such as children with neurodevelopmental disorders experience a variety of barriers and limitations to daily activities that may cause them to engage in less active and more sedentary lifestyles than their typically developing (TD) peers (Barnett et al., 2013; Papadopoulos et al., 2020). This is of particular concern given that this population is at an increased risk of physical comorbidities (King-Dowling et al., 2019). Developmental Coordination Disorder (DCD) is a neurodevelopmental disorder that affects approximately $2-6 \%$ of children (American Psychiatric Association, 2013; Lingam et al., 2009) and has received considerable attention regarding patterns of movement behaviours during childhood, but work in this area has focused largely on MVPA. For instance, a systematic review of the literature found 


\section{MOVEMENT COMPOSITIONS AND MENTAL HEALTH}

children with DCD engage in lower levels of MVPA than TD children and these differences persist as they age into adolescence (Rivilis et al., 2011). While there has been little research on the early years, recent work using accelerometry suggests that preschool-aged children at risk for DCD (rDCD) engage in similar amounts of MVPA, LPA and sedentary time compared to their TD peers (King-Dowling et al., 2019). However, studies taking a more comprehensive approach that moves beyond considering absolute amounts of time engaging in each behaviour to instead focus on the relative proportion of time spent engaging in each behaviour within a finite window have yet to be conducted.

To date, very few studies have taken an integrative approach to investigate associations between movement behaviours and health outcomes among preschool children. Even fewer studies have adopted compositional data analysis methods to examine how time spent engaging in one movement behaviour relative to the others or reallocation of time between movement behaviours is related to healthy development. Recent work using accelerometry has shown that 24-hour movement compositions significantly predict fundamental motor skills (Kuzik et al., 2020; Mota et al., 2020) and cognitive development (Bezerra et al., 2021; Kuzik et al., 2020) in preschool-age children. Although evidence has demonstrated beneficial associations between different intensities of physical activity and indicators of mental health within samples of children and youth between the ages of 6 to 17 years (Carson et al., 2016; Dumuid, Maher, et al., 2018; Fairclough et al., 2021), the only study to examine these relationships among preschoolage children observed null relationships (Kuzik et al., 2020). Combined with the fact that children at risk for DCD experience higher rates of mental health problems beginning as early as the preschool years (Rodriguez et al., 2019), this dearth of research highlights the need for additional work - particularly among larger and more diverse samples - to better understand 


\section{MOVEMENT COMPOSITIONS AND MENTAL HEALTH}

whether movement behaviours influence mental health in the early years. Although existing literature has examined different pathways by which physical activity and sedentary time influence indicators of mental health among children classified as rDCD (Bulten et al., 2020; Li et al., 2021), studies adopting novel techniques to investigate how the interplay between these movement behaviours affects their mental health have yet to be conducted.

Therefore, the purpose of the present study is twofold: 1) examine whether differences in wake-time movement compositions (i.e., sedentary time, LPA, MVPA) exist between preschool children classified as TD and rDCD; and 2) investigate associations between the movement composition of preschool children and indicators of mental health.

\section{Methods}

\section{Participants}

The present study is cross-sectional, utilizing baseline cohort data from the Coordination and Activity Tracking in $\mathrm{CHildren}(\mathrm{CATCH})$ study. $\mathrm{CATCH}$ is a prospective cohort study examining the relationships among motor coordination, physical fitness, physical activity, and

health outcomes beginning in early childhood (Cairney et al., 2015). Children 4 to 5 years of age were screened for poor motor coordination during several stages of the recruitment process to achieve a target sample size of 300 TD children and 300 children classified as rDCD. Children with a physical disability or a medical condition that affected their motor coordination (e.g. cerebral palsy) were not eligible for the study, in addition to children with a birth weight lower than $1500 \mathrm{~g}$, to rule out conditions other than DCD that may account for children's poor motor coordination. Additional details of the study design and recruitment procedures are available elsewhere (Cairney et al., 2015, 2019). Informed, written consent was provided by the 
MOVEMENT COMPOSITIONS AND MENTAL HEALTH

parents/guardians of all participants. The CATCH study protocol was approved by an institutional research ethics board.

\section{Measures}

Demographics. Parents reported their total household income as well as their child's race/ethnicity and sex. Total household income was coded into four groups: less than $\$ 50,000$, between $\$ 50,000$ and $\$ 99,999$, between $\$ 100,000$ and $\$ 149,999$, and greater than $\$ 150,000$. Race/ethnicity was coded into two groups: White, and non-White, which included Asian, Middle Eastern, Black, Indigenous, Latino and Mixed/Other.

Anthropometric data. Height and weight were obtained using a calibrated weigh scale and stadiometer and used to calculate body mass index $\left(\operatorname{mass}(\mathrm{kg}) / \mathrm{height}(\mathrm{m})^{2}\right) \mathrm{z}$-scores based on age and sex as per the Center for Disease Control growth charts (Kuczmarski et al., 2002).

Developmental Coordination Disorder Risk. The Movement Assessment Battery for Children $2^{\text {nd }}$ Edition (MABC-2) is a standardized test consisting of 8 items categorized into 3 groups: (1) manual dexterity, (2) aiming and catching, and (3) static and dynamic balance. Children's raw scores on these items are converted into standardized scores based on age and then further classified into overall percentile ranks. The MABC-2 is widely used for the identification of DCD and has been shown to be valid and reliable for assessing poor motor coordination in children ages 3 to 5 years (Ellinoudis et al., 2011). The tool has also been identified as the criterion standard for assessing DCD in children (Blank et al., 2019). In the current study, children who scored above the $16^{\text {th }}$ percentile were classified as TD, and children who scored at or below the $16^{\text {th }}$ percentile were classified as at risk for DCD (Blank et al., 2012). All MABC-2 assessments were administered and scored by trained research assistants. As noted earlier, the 
MOVEMENT COMPOSITIONS AND MENTAL HEALTH

design of the CATCH study also involved exclusion criteria pertaining to conditions that may otherwise explain a child's poor motor coordination and thus result in them being classified as rDCD for extraneous reasons.

Movement Behaviours. Accelerometry is a well-established device-assessed measure of physical activity and sedentary time in children (Pate et al., 2010). Children's wake-time movement behaviours - sedentary time, LPA, and MVPA - were assessed using an Actigraph GT3X+ activity monitor device worn on their right hip secured by a belt around their waist for 7 days. The device was only removed for periods of sleep time and prolonged water exposure. Parents were asked to keep a diary to record when the accelerometer was put on and taken off. Minimum wear time criteria for including a child's accelerometer data were set at three days with a minimum of ten hours per day wear time. Given that young children are inclined toward short bursts of activity, data were analyzed in three-second epochs using ActiLife 6 software (ActiGraph, Pensacola, FL) to allow for a more accurate representation of activity levels (Green et al., 2011; Obeid et al., 2011; Pate et al., 2006). The 2008 Evenson activity cut point for children was used to determine time spent in sedentary behaviour (in minutes per day), as well as time spent engaging in light, and moderate-to-vigorous physical activity (in minutes per day). These cut points have been previously validated in children ages 5 to 8 (Trost et al., 2000).

Internalizing and Externalizing Problems. Parents completed the Child Behavior Checklist (CBCL) for ages 1.5 to 5 years during baseline testing for the CATCH study (Achenbach, 2011). The CBCL is a validated and reliable tool commonly used to assess externalizing and internalizing problems, among children. The test-retest reliability of the CBCL scale scores range from 0.68 to 0.92 and reported classification accuracy has been shown to range from 74-84\% (Achenbach \& Rescorla, 2013). In the present study, we used the DSM-IV 


\section{MOVEMENT COMPOSITIONS AND MENTAL HEALTH}

broad band internalizing and externalizing problems scales. The internalizing problems scale score represents the sum of the emotionally reactive, anxious/depressed, withdrawn, and somatic complaints syndrome subscales, whereas the externalizing problems scale score represents the attention problems and aggressive behaviour syndrome subscales. High levels of validity and reliability of the DSM-IV oriented scales have been established (Achenbach et al., 2003b), and extensive normative data are available for children between the ages of 1.5 to 5 years. Internal consistency for the DSM-IV orientated scales has been reported at 0.75 , with a test-retest reliability of 0.83 (Achenbach et al., 2003). Although DSM-V oriented scales have since been created, the values for these scales were considerably right skewed, and various transformations did not improve the distribution of the data; therefore, the standardized t-score DSM-IV scales were used. The CBCL DSM-IV standardized t-scores represent a comparison of a child's raw score to what would be a typical score reported for their gender and age based on a normative sample. These scores are scaled so that 50 is the mean for the child's age and gender, with a standard deviation of 10 points and higher scores representing greater problems.

\section{Data Analysis}

All analyses were performed in R (Version 4.0.3) and R Studio (Version 1.3.1093). First, we inspected the data for missingness using the mice package (van Buuren \& GroothuisOudshoorn, 2011). Data were considered missing at random and multiple imputation by chained equations was conducted using the mice package to replace missing values. However, the packages used for subsequent compositional analyses cannot handle multiply imputed datasets, and therefore, one of the five imputed datasets was randomly selected for the purpose of our analyses. 


\section{MOVEMENT COMPOSITIONS AND MENTAL HEALTH}

Next, we conducted the primary data analyses, which consisted of (1) comparing waketime movement compositions between children classified as TD and rDCD, and (2) investigating the relationship between the movement composition and indicators of mental health, and the influence that changes to the movement composition may impart for mental health among preschool children.

The compositions (van den Boogaart \& Tolosana-Delgado, 2008), and deltacomp (Stanford, 2020) packages were used for all compositional data analyses. Wake-time movement behaviours (sedentary time, LPA, MVPA) assessed via device-based measures such as accelerometry are mutually exclusive and exhaustive parts of the movement composition. Collectively, time spent in each of these behaviours must sum to the entire wake-time period over the course of a day. Simply stated, the amount of time an individual engages in one movement behaviour cannot be changed without an equivalent change among the other behaviours. For example, reducing sedentary time would mean an individual spends more time engaging in LPA or MVPA. Compared to previous work that has commonly reported movement behaviours such as physical activity in absolute terms, movement compositions are relative data and therefore take into account the codependence amongst all behaviours that make up the composition. Due to potential issues with multicollinearity, however, there are statistical challenges with including all movement behaviours within a single model using traditional statistical methodologies (Dumuid, Stanford, et al., 2018). Compositional data analysis techniques can be used to address this limitation (Chastin et al., 2015). Compositional data analysis involves expressing time spent engaging in different movement behaviours during a finite period in relative terms, as a set of isometric log-ratio (ilr) coordinates (Aitchison, 1982). 
MOVEMENT COMPOSITIONS AND MENTAL HEALTH

Given that ilrs cannot be computed if there are zeros in the data that represents the relative time spent in each movement behaviour, the first step in our analysis involved checking for zero values across the three parts (sedentary time, LPA, MVPA) of our daily wake-time composition. Once we confirmed none were present, we created the ilr coordinates using a sequential binary partition process (Egozcue \& Pawlowsky-Glahn, 2005). Computing the first pivot coordinate to provide an ilr involved partitioning the composition by coding time spent in one behaviour as the numerator $(+1)$ in the equation, and time amongst the other two behaviours coded as the denominator (-1). After partitioning out the first behaviour, it is coded as an uninvolved part (0) in the sequential binary partition when creating the second pivot coordinate. Time spent engaging in the remaining two behaviours were then coded to be in the numerator $(+1)$ and the denominator $(-1)$. The pivot coordinates were used to produce the ilrs that express the movement composition. To illustrate this process with an example, the first coordinate may include all relative information regarding sedentary time versus the geometric mean of time spent engaging in any intensity of physical activity, and the second coordinate may represent LPA versus MVPA. Following this example, ilr transformations of the movement behaviour composition were expressed using the following equations:

$$
\begin{aligned}
& \text { (1) } i l r_{1}^{(S B)}=\sqrt{\frac{2}{3}} \ln \frac{S B}{\sqrt{L I P A \times M V P A}} \\
& \text { (2) } i \operatorname{lr}_{2}^{(S B)}=\frac{1}{\sqrt{2}} \ln \frac{L I P A}{M V P A}
\end{aligned}
$$

The final ilrs provided geometric means of all parts, which were linearly adjusted to sum to 1 , or $100 \%$ of the wake-time period, with each movement behaviour (i.e., part) expressed as a proportion of the whole composition. We closed our composition to 724 mins ( $12 \mathrm{hr}$ ), which represented the mean daily wear time for the sample. Given that wear time varies across 


\section{MOVEMENT COMPOSITIONS AND MENTAL HEALTH}

participants, this means that the amount of time each participant spent engaging in each movement behaviour was adjusted to a wake-time window of 724 mins.

To answer the first research question of whether differences in mean time-use movement compositions exist between preschool children classified as TD and rDCD, a compositional multivariate analysis of covariance model was computed. Wake time movement behaviours (sedentary time, LPA, MVPA) were included as the primary variables of interest, rDCD status (yes/no) was set as the grouping variable, and the model was adjusted for covariates (age, sex, socioeconomic status, ethnicity). Models with each movement behaviour set as the dominant activity in the model provide equivalent estimates, therefore, only one set of ilr coordinates were constructed, with sedentary time set as the dominant activity.

For the second research question regarding whether movement compositions impact indicators of mental health among preschool children, we pooled the subsamples of children with TD and rDCD given the similarity in their movement compositions. Three sets of ilrs were constructed, with each set treating a different movement behaviour as the primary variable of interest. The ilrs were used to compute a series of linear regression models examining associations between each movement behaviour and the indicators of mental health. The regression coefficients and standard errors for the first ilr coordinate for sedentary time, LPA, and MVPA are presented, considering the focus is on time spent in each behaviour relative to the remaining two behaviours. Each model was adjusted for rDCD status, age, sex, socioeconomic status, race/ethnicity and body mass index percentile. Assumptions for linearity, homogeneity and normality were examined using the performance package (Lüdecke et al., 2021). All assumptions were satisfied for each model, although a total of $27(4.6 \%)$ and $24(4.1 \%)$ influential observations were identified for the internalizing and externalizing problems models, 
MOVEMENT COMPOSITIONS AND MENTAL HEALTH

respectively, as per Cook's distance values greater than $4 / n$. Ordinary least squares regression estimates are sensitive to highly influential observations, therefore, robust regression was employed using the robustbase package to reduce the influence of these observations (Maechler et al., 2021).

Finally, compositional isotemporal substitution modeling was performed to examine the impact on internalizing and externalizing problems of reallocating 5, 10, 15, 20 and 25 min in one movement behaviour to another (e.g., replacing 5 min of sedentary time with 5 min of LPA). These models followed the methods outlined by Dumuid et al. (2019) and were computed using the deltacomp package (Stanford, 2020). This analysis involved creating a series of new movement compositions to reflect the range of $5 \mathrm{~min}$ isotemporal substitution increments for each movement behaviour contrast (sedentary time vs. LPA; sedentary time vs. MVPA; LPA vs. MVPA), with the base regression model set as the starting composition. Statistical significance for all analyses was set at $\alpha<.05$.

\section{Results}

\section{Data Inspection}

Inspection of the data revealed ten (1.7\%) missing values for ethnicity, 17 (2.9\%) for total household income, and one $(0.2 \%)$ for the MABC-2. A total of 75 participants $(12.7 \%)$ had missing accelerometry data, and seven participants $(1.2 \%)$ had missing mental health data. Participants with missing accelerometry data had significantly higher body mass index percentile values as well as lower household income and MABC-2 scores. Participants with missing mental health data had significantly lower household income. For these reasons, data was considered missing at random and missing values were replaced via stochastic regression imputation.

\section{Sample Demographics}


MOVEMENT COMPOSITIONS AND MENTAL HEALTH

On average, participants in the present study were 4.94 years of age and the sample consisted of slightly more than half boys $(57.4 \%)$, were identified primarily as White $(81.0 \%)$ and lived in households with a total income of more than $\$ 50,000(86.8 \%)$. There were more children identified as typically developing $(n=301,51.1 \%)$ than those classified as $\operatorname{rDCD}(n=$ 288, 48.9\%). Descriptive statistics for demographic variables, indicators of mental health as well as absolute and relative time spent engaging in each movement behaviour are presented for the full sample, and by group, in Table 1.

\section{Group Differences in Movement Compositions Based on rDCD Status}

The results of our compositional multivariate analysis of covariance testing whether movement compositions differed between preschool children classified as TD and rDCD failed to reveal significant differences in their mean daily time use patterns after adjusting for covariates, $F(2,580)=0.56, p=0.57, \Lambda=1.00$. The relative distribution of time spent engaging in sedentary time, LPA and MVPA for each group is presented in Figure 1 as the log-ratio differences between the group compositional mean and the full sample compositional mean after centering the data. The scatterplot distributions of the group compositions are shown in separate ternary diagrams (Figure 2A-B).

\section{Associations Between Movement Compositions and Indicators of Mental Health}

Given the similarity in movement compositions for TD and rDCD children, we pooled the groups and investigated associations with mental health using the full sample. The results of our regression models demonstrated preschool-age children's movement compositions explained $3 \%$ of the variance for internalizing problems (Adjusted $\mathrm{R}^{2}=0.03$ ) and $8 \%$ for externalizing problems (Adjusted $\mathrm{R}^{2}=0.08$ ). Within the compositional linear regression models, a significant negative association was found when predicting externalizing problems from sedentary time $(p=$ 


\section{MOVEMENT COMPOSITIONS AND MENTAL HEALTH}

0.03), however, significant associations were not observed for the other five relationships (all $p$ 's $>$.05; Table 2).

Isotemporal substitution modeling results are presented graphically in Figure 3.

Reallocating 5, 10, 15, 20 and 25 min of time between each of the movement behaviours was not found to impact internalizing problems (all $p s>0.05$ ). This was to be expected given the base regression model failed to show a significant association between any one movement behaviour (relative to the others) and internalizing problems. Contrary to our base regression model, we failed to observe a significant influence on externalizing problems when time spent engaging in any one movement behaviour was replaced with 5, 10, 15, 20 and 25 min of time engaging in another movement behaviour (all ps > 0.05).

\section{Discussion}

This study was the first to examine whether wake-time movement compositions differ between preschool children classified as TD and rDCD, and represents the largest study to investigate the impact of wake-time movement behaviours on indicators of mental health among preschool children using accelerometry. Our results indicated that movement compositions were relatively similar for TD preschool-age children and those classified as rDCD; with both groups, on average, engaging in nearly five hours of activity of varying intensities during a 12-hour wake period. Given the similarity in movement compositions in children with TD and rDCD, we pooled the sample and adjusted for rDCD status when investigating associations between sedentary time, LPA and MVPA with indicators of mental health. Findings demonstrated a significant negative relationship for sedentary time (relative to time in LPA and MVPA) and externalizing problems, whereas null associations were observed for each of the other relationships. Collectively, these findings suggest preschool children engage in a considerable 
MOVEMENT COMPOSITIONS AND MENTAL HEALTH

amount of activity across the course of a day, but their movement behaviour patterns explain very little variance in parent-reported measures of internalizing and externalizing problems.

Previous research has generally suggested that children with DCD engage in less active and more sedentary lifestyles than their TD peers (Rivilis et al., 2011). This study was the first to use compositional data analysis techniques to determine whether these traditionally observed differences in movement behaviours are apparent as early as the preschool years. Using accelerometry, our findings showed that the relative amount of time spent engaging in sedentary time, LPA and MVPA across the course of an average day was roughly equivalent for TD children and those classified as rDCD. Although the present study focused on general patterns of movement behaviours, other work using the baseline CATCH cohort suggests that there may be subtle differences in how preschool children classified as rDCD accumulate their MVPA (KingDowling et al., 2019). Given that the CATCH study will track this sample into late childhood, future work will provide a better understanding of when differences in movement compositions begin to manifest. For instance, it is reasonable to hypothesize that children classified as rDCD will become less active and more sedentary when they reach middle childhood, as this period represents when many children begin playing organized sports and engaging in other active pursuits that require greater motor demands. For children with DCD, their poor motor skills may affect their confidence to participate (Cairney et al., 2005), thus leading them to engage in more sedentary pursuits. Fortunately, multiple waves of data from the CATCH study will be able to answer many of the outstanding questions in this area.

Beyond examining potential differences in movement compositions among children classified as TD and rDCD during the preschool years, the present study also contributes to the dearth of literature investigating the impact of movement behaviours on mental health during the 


\section{MOVEMENT COMPOSITIONS AND MENTAL HEALTH}

early years. For the most part, our findings align with previous research that has failed to demonstrate significant associations between wake-time movement behaviours and mental health problems (Kuzik et al., 2020). In fact, wake-time movement compositions appear to explain only a very small amount of the variance in internalizing (3\%) and externalizing (8\%) problems during this early life stage. From this perspective, it may not be surprising that we found replacing up to 25 mins of sedentary time with either LPA or MVPA confers negligible benefits for mental health. Although recent cross-sectional studies have shown movement compositions are positively associated with important physical and cognitive developmental outcomes in the early years (Bezerra et al., 2021; Kuzik et al., 2020; Mota et al., 2020), the benefits for mental health and wellbeing may not manifest until children are of school age (Fairclough et al., 2021). Despite these findings, it is worth noting that accelerometry data does not provide contextspecific information about the activities people are engaging in; and therefore, recent advances in machine learning approaches for classifying device-measured movement behaviours may provide novel insights about the complexity of these relationships (Ahmadi et al., 2020).

Although five out of the six relationships between movement behaviours and indicators of mental health were not found to be significant, a significant negative association was observed for sedentary time and externalizing problems. One potential explanation for this finding relates to measurement, not only related to the CBCL, but also accelerometry. Within the attention problems subscale (which together with the aggressive behaviour subscale make up the externalizing problems scale) are items that may have implications for classification of accelerometer-based movements. For example, one item asks about whether a child cannot sit still. Given that hip worn accelerometers cannot distinguish between sedentary postures (i.e., sitting versus standing) (Kurita et al., 2017), it is a distinct possibility that sedentary time is 
MOVEMENT COMPOSITIONS AND MENTAL HEALTH

misclassified as LPA if a child is constantly fidgeting in their seat or while they stand. For children who score high on measures of attention problems and/or may be diagnosed with ADD/ADHD - a common co-morbidity for children diagnosed with DCD (Kadesjo \& Gillberg, 1999) - these instances would serve to strengthen negative associations between sedentary time and externalizing problems. Contrary to this argument, however, is that these children may be expending $\geq 1 \mathrm{MET}$ and these movement are therefore accurately captured. Moving forward, postural data obtained from wearables that measure posture may help to improve the classification of lower intensity movement behaviours, particularly among individuals with inattention problems.

From a public health standpoint, our findings suggest that movement behaviour-related interventions may have limited efficacy when seeking to reduce mental health problems among preschool children. This is not to say that interventions focused on improving movement compositions should be avoided, as they have the potential to provide important benefits for cardiometabolic health and cognitive function (Bezerra et al., 2021; Kuzik et al., 2020; Mota et al., 2020), but rather, that individuals involved in developing health promotion strategies and campaigns for preschool children should be aware that effects for emotional and behavioural outcomes may be negligible. Until researchers begin employing methodologies that provide contextual information about what types of activities preschool children engage in during their sedentary time (i.e., mentally active vs. passive; Hallgren et al., 2020) or while active, in addition to who they engage in these activities with, we will only have a gross understanding of associations between movement compositions and mental health. Implementing such methodologies with young children may be fraught with challenges though. 


\section{MOVEMENT COMPOSITIONS AND MENTAL HEALTH}

Although the present paper addresses gaps in the existing literature, it is not without limitations. First, movement behaviours are traditionally examined using the 24-hour paradigm, which also includes sleep. However, data pertaining to sleep duration was not collected in the CATCH study; thus, some of the variance in mental health problems explained by sleep was not captured. Second, the sample consisted of primarily White children living in households with a total income above the poverty line, which may potentially limit the generalizability of our findings to the broader Canadian population, although time spent engaging in each movement behaviour was similar to that observed among a nationally representative sample of Canadian preschool children (Carson, Tremblay, et al., 2017). Finally, this study involved cross-sectional data, which limits our ability to make causal inferences about the directionality of the relationships between movement compositions and mental health.

In conclusion, we showed that wake-time movement compositions were similar among preschool children classified as TD and rDCD. These findings indicate that differences in sedentary time and physical activity typically observed between these groups likely develop later in childhood. Furthermore, findings revealed that at this young age, the overall composition of wake-time movement behaviours does not appear to play an influential role for children's mental health. While movement behaviour-related interventions may have beneficial effects for other important developmental outcomes, interventionists should be aware that the impact on mental health may be trivial. 


\section{Notes}

CRediT Author Statement. Denver Brown: Conceptualization, Methodology, Writing - original draft, Formal analysis, Data curation, Visualization. Matthew Kwan: Methodology, Writing review and editing, Supervision, Funding acquisition. Sara King-Dowling: Writing - review and editing. John Cairney: Methodology, Writing - review and editing, Supervision, Funding acquisition.

Conflicts of interest: None

Funding: The Coordination and Activity Tracking in Children (CATCH) study was funded by the Canadian Institutes of Health Research (CIHR Award \#: MOP 126015). 
MOVEMENT COMPOSITIONS AND MENTAL HEALTH

\section{References}

Achenbach, T. M. (2011). Child Behavior Checklist. In Encyclopedia of Clinical Neuropsychology (pp. 546-552). Springer New York. https://doi.org/10.1007/978-0-38779948-3_1529

Achenbach, T. M., Dumenci, L., \& Rescorla, L. A. (2003). DSM-oriented and empirically based approaches to constructing scales from the same item pools. Journal of Clinical Child \& Adolescent Psychology, 32(3), 328-340.

https://doi.org/10.1207/S15374424JCCP3203_02

Achenbach, T., \& Rescorla, L. (2013). Achenbach System of Empirically Based Assessment. In Encyclopedia of Autism Spectrum Disorders (pp. 31-39). Springer: New York, NY. https://doi.org/10.1007/978-1-4419-1698-3_219

Ahmadi, M. N., Pavey, T. G., \& Trost, S. G. (2020). Machine learning models for classifying physical activity in free-living preschool children. Sensors (Basel, Switzerland), 20(16), 4364. https://doi.org/10.3390/s20164364

Aitchison, J. (1982). The statistical analysis of compositional data. Journal of the Royal Statistical Society. Series B (Methodological), 44(2), 139-177.

American Psychiatric Association. (2013). Diagnostic and Statistical Manual of Mental Disorders (Fifth Edition). American Psychiatric Association. https://doi.org/10.1176/appi.books.9780890425596

Barnett, A. L., Dawes, H., \& Wilmut, K. (2013). Constraints and facilitators to participation in physical activity in teenagers with Developmental Co-ordination Disorder: An exploratory interview study. Child: Care, Health and Development, 39(3), 393-403. https://doi.org/10.1111/j.1365-2214.2012.01376.x 
Bezerra, T. A., Clark, C. C. T., Souza Filho, A. N. D., Fortes, L. D. S., Mota, J. A. P. S., Duncan, M. J., \& Martins, C. M. D. L. (2021). 24-hour movement behaviour and executive function in preschoolers: A compositional and isotemporal reallocation analysis. European Journal of Sport Science, 21, 1064-1072. https://doi.org/10.1080/17461391.2020.1795274

Blank, R., Barnett, A. L., Cairney, J., Green, D., Kirby, A., Polatajko, H., Rosenblum, S., SmitsEngelsman, B., Sugden, D., Wilson, P., \& Vinçon, S. (2019). International clinical practice recommendations on the definition, diagnosis, assessment, intervention, and psychosocial aspects of developmental coordination disorder. Developmental Medicine \& Child Neurology, 61(3), 242-285. https://doi.org/10.1111/dmcn.14132

Blank, R., Smits-Engelsman, B., Polatajko, H., \& Wilson, P. (2012). European Academy for Childhood Disability (EACD): Recommendations on the definition, diagnosis and intervention of developmental coordination disorder (long version). Developmental Medicine and Child Neurology, 54(1), 54-93. https://doi.org/10.1111/j.14698749.2011.04171.x

Bulten, R., Brown, D., Rodriguez, C., \& Cairney, J. (2020). Association of sedentary behaviour on internalizing problems in children with and without motor coordination problems. Mental Health and Physical Activity, 18, 100325. https://doi.org/10.1016/j.mhpa.2020.100325

Cairney, J., Hay, J. A., Faught, B. E., Wade, T. J., Corna, L., \& Flouris, A. (2005).

Developmental Coordination Disorder, generalized self-efficacy toward physical activity, and participation in organized and free play activities. Journal of Pediatrics, 147(4), 515520. https://doi.org/10.1016/j.jpeds.2005.05.013 


\section{MOVEMENT COMPOSITIONS AND MENTAL HEALTH}

Cairney, J., Missiuna, C., Timmons, B. W., Rodriguez, C., Veldhuizen, S., King-Dowling, S., Wellman, S., \& Le, T. (2015). The Coordination and Activity Tracking in CHildren (CATCH) study: Rationale and design. BMC Public Health, 15(1), 1266. https://doi.org/10.1186/s12889-015-2582-8

Cairney, J., Veldhuizen, S., Rodriguez, M. C., King-Dowling, S., Kwan, M. Y., Wade, T., Price, D., Missiuna, C., \& Timmons, B. (2019). Cohort profile: The Canadian coordination and activity tracking in children (CATCH) longitudinal cohort. BMJ Open, 9(9), e029784. https://doi.org/10.1136/bmjopen-2019-029784

Carson, V., Lee, E.-Y., Hewitt, L., Jennings, C., Hunter, S., Kuzik, N., Stearns, J. A., Unrau, S. P., Poitras, V. J., Gray, C., Adamo, K. B., Janssen, I., Okely, A. D., Spence, J. C., Timmons, B. W., Sampson, M., \& Tremblay, M. S. (2017). Systematic review of the relationships between physical activity and health indicators in the early years $(0-$ 4 years). BMC Public Health, 17(5), 854. https://doi.org/10.1186/s12889-017-4860-0

Carson, V., Tremblay, M. S., Chaput, J.-P., \& Chastin, S. F. M. (2016). Associations between sleep duration, sedentary time, physical activity, and health indicators among Canadian children and youth using compositional analyses. Applied Physiology, Nutrition, and Metabolism, 41(6 Suppl 3), S294-302. https://doi.org/10.1139/apnm-2016-0026

Carson, V., Tremblay, M. S., \& Chastin, S. F. M. (2017). Cross-sectional associations between sleep duration, sedentary time, physical activity, and adiposity indicators among Canadian preschool-aged children using compositional analyses. BMC Public Health, 17(5), 123-131. https://doi.org/10.1186/s12889-017-4852-0

Chastin, S. F. M., Palarea-Albaladejo, J., Dontje, M. L., \& Skelton, D. A. (2015). Combined effects of time spent in physical activity, sedentary behaviors and sleep on obesity and 


\section{MOVEMENT COMPOSITIONS AND MENTAL HEALTH}

cardio-metabolic health markers: A novel compositional data analysis approach. PLOS ONE, 10, e0139984. https://doi.org/10.1371/journal.pone.0139984

Dumuid, D., Maher, C., Lewis, L. K., Stanford, T. E., Martín Fernández, J. A., Ratcliffe, J., Katzmarzyk, P. T., Barreira, T. V., Chaput, J.-P., Fogelholm, M., Hu, G., Maia, J., Sarmiento, O. L., Standage, M., Tremblay, M. S., Tudor-Locke, C., \& Olds, T. (2018). Human development index, children's health-related quality of life and movement behaviors: A compositional data analysis. Quality of Life Research, 27(6), 1473-1482. https://doi.org/10.1007/s11136-018-1791-x

Dumuid, D., Pedišić, Ž., Stanford, T. E., Martín-Fernández, J.-A., Hron, K., Maher, C. A., Lewis, L. K., \& Olds, T. (2019). The compositional isotemporal substitution model: A method for estimating changes in a health outcome for reallocation of time between sleep, physical activity and sedentary behaviour. Statistical Methods in Medical Research, 28(3), 846-857. https://doi.org/10.1177/0962280217737805

Dumuid, D., Stanford, T. E., Martin-Fernández, J.-A., Pedišić, Ž., Maher, C. A., Lewis, L. K., Hron, K., Katzmarzyk, P. T., Chaput, J.-P., Fogelholm, M., Hu, G., Lambert, E. V., Maia, J., Sarmiento, O. L., Standage, M., Barreira, T. V., Broyles, S. T., Tudor-Locke, C., Tremblay, M. S., \& Olds, T. (2018). Compositional data analysis for physical activity, sedentary time and sleep research. Statistical Methods in Medical Research, 27, 37263738. https://doi.org/10.1177/0962280217710835

Egozcue, J. J., \& Pawlowsky-Glahn, V. (2005). Groups of parts and their balances in compositional data analysis. Mathematical Geology, 37(7), 795-828. https://doi.org/10.1007/s11004-005-7381-9 


\section{MOVEMENT COMPOSITIONS AND MENTAL HEALTH}

Ellinoudis, T., Evaggelinou, C., Kourtessis, T., Konstantinidou, Z., Venetsanou, F., \& Kambas, A. (2011). Reliability and validity of age band 1 of the Movement Assessment Battery for Children-Second Edition. Research in Developmental Disabilities, 32(3), 1046-1051. https://doi.org/10.1016/j.ridd.2011.01.035

Fairclough, S. J., Tyler, R., Dainty, J. R., Dumuid, D., Richardson, C., Shepstone, L., \& Atkin, A. J. (2021). Cross-sectional associations between 24-hour activity behaviours and mental health indicators in children and adolescents: A compositional data analysis. Journal of Sports Sciences, 39, 1602-1614. https://doi.org/10.1080/02640414.2021.1890351

Feng, J., Zheng, C., Sit, C. H.-P., Reilly, J. J., \& Huang, W. Y. (2021). Associations between meeting 24-hour movement guidelines and health in the early years: A systematic review and meta-analysis. Journal of Sports Sciences, O(0), 1-13. https://doi.org/10.1080/02640414.2021.1945183

Green, D., Lingam, R., Mattocks, C., Riddoch, C., Ness, A., \& Emond, A. (2011). The risk of reduced physical activity in children with probable Developmental Coordination Disorder: A prospective longitudinal study. Research in Developmental Disabilities, 32(4), 1332-1342. https://doi.org/10.1016/j.ridd.2011.01.040

Hallgren, M., Dunstan, D. W., \& Owen, N. (2020). Passive versus mentally active sedentary behaviors and depression. Exercise and Sport Sciences Reviews, 48(1), 20-27. https://doi.org/10.1249/JES.0000000000000211

Kadesjo, B., \& Gillberg, C. (1999). Developmental Coordination Disorder in Swedish 7-year-old children. Journal of the American Academy of Child \& Adolescent Psychiatry, 38(7), 820-828. https://doi.org/10.1097/00004583-199907000-00011 
MOVEMENT COMPOSITIONS AND MENTAL HEALTH

King-Dowling, S., Kwan, M. Y. W., Rodriguez, C., Missiuna, C., Timmons, B. W., \& Cairney, J. (2019). Physical activity in young children at risk for developmental coordination disorder. Developmental Medicine \& Child Neurology, 61(11), 1302-1308. https://doi.org/10.1111/dmcn.14237

King-Dowling, S., Proudfoot, N. A., \& Obeid, J. (2019). Comorbidity among chronic physical health conditions and neurodevelopmental disorders in childhood. Current Developmental Disorders Reports, 6(4), 248-258. https://doi.org/10.1007/s40474-01900173-x

Kuczmarski, R. J., Ogden, C. L., Guo, S. S., Grummer-Strawn, L. M., Flegal, K. M., Mei, Z., Wei, R., Curtin, L. R., Roche, A. F., \& Johnson, C. L. (2002). 2000 CDC Growth Charts for the United States: Methods and development. Vital and Health Statistics. Series 11, Data from the National Health Survey, 246, 1-190.

Kurita, S., Yano, S., Ishii, K., Shibata, A., Sasai, H., Nakata, Y., Fukushima, N., Inoue, S., Tanaka, S., Sugiyama, T., Owen, N., \& Oka, K. (2017). Comparability of activity monitors used in Asian and Western-country studies for assessing free-living sedentary behaviour. PLOS ONE, 12(10), e0186523. https://doi.org/10.1371/journal.pone.0186523

Kuzik, N., Naylor, P.-J., Spence, J. C., \& Carson, V. (2020). Movement behaviours and physical, cognitive, and social-emotional development in preschool-aged children: Cross-sectional associations using compositional analyses. PLOS ONE, 15(8), e0237945. https://doi.org/10.1371/journal.pone.0237945

Li, Y.-C., Kwan, M. Y. W., King-Dowling, S., Rodriguez, M. C., \& Cairney, J. (2021). Does physical activity and BMI mediate the association between DCD and internalizing 


\section{MOVEMENT COMPOSITIONS AND MENTAL HEALTH}

problems in early childhood? A partial test of the Environmental Stress Hypothesis. Human Movement Science, 75, 102744. https://doi.org/10.1016/j.humov.2020.102744

Lingam, R., Hunt, L., Golding, J., Jongmans, M., \& Emond, A. (2009). Prevalence of Developmental Coordination Disorder using the DSM-IV at 7 years of age: A UK population-based study. Pediatrics, 123(4), e693-e700. https://doi.org/10.1542/peds.2008-1770

Lüdecke, D., Mattan S. Ben-Shachar, Indrajeet Patil, Philip Waggoner, \& Dominique Makowski. (2021). Assessment of regression models performance. Journal of Open Source Software, 6, 3139. https://doi.org/10.21105/joss.03139

Maechler, M., Rousseeuw, P., Croux, C., Todorov, V., Ruckstuhl, A., Salibian-Barrera, M., Verbeke, T., Koller, M., Conceicao, E. L., \& Anna di Palma, M. (2021). robustbase: Basic robust statistics (R package version 0.93-7) [Computer software]. http://robustbase.r-forge.r-project.org/

Mota, J. G., Clark, C. C. T., Bezerra, T. A., Lemos, L., Reuter, C. P., Mota, J. A. P. S., Duncan, M. J., \& Martins, C. M. D. L. (2020). Twenty-four-hour movement behaviours and fundamental movement skills in preschool children: A compositional and isotemporal substitution analysis. Journal of Sports Sciences, 38(18), 2071-2079. https://doi.org/10.1080/02640414.2020.1770415

Obeid, J., Nguyen, T., Gabel, L., \& Timmons, B. W. (2011). Physical activity in Ontario preschoolers: Prevalence and measurement issues. Applied Physiology, Nutrition, and Metabolism, 36, 291-297. https://doi.org/10.1139/h11-002

Papadopoulos, N. V., Whelan, M., Skouteris, H., Williams, K., McGinley, J., Shih, S. T. F., Emonson, C., Moss, S. A., Sivaratnam, C., Whitehouse, A. J. O., \& Rinehart, N. J. 


\section{MOVEMENT COMPOSITIONS AND MENTAL HEALTH}

(2020). An examination of parent-reported facilitators and barriers to organized physical activity engagement for youth with neurodevelopmental disorders, physical, and medical conditions. Frontiers in Psychology, 11, 2358. https://doi.org/10.3389/fpsyg.2020.568723

Pate, R. R., Almeida, M. J., McIver, K. L., Pfeiffer, K. A., \& Dowda, M. (2006). Validation and calibration of an accelerometer in preschool children. Obesity, 14(11), 2000-2006. https://doi.org/10.1038/oby.2006.234

Pate, R. R., O’Neill, J. R., \& Mitchell, J. (2010). Measurement of physical activity in preschool children. Medicine and Science in Sports and Exercise, 42(3), 508-512. https://doi.org/10.1249/MSS.0b013e3181cea116

Rivilis, I., Hay, J., Cairney, J., Klentrou, P., Liu, J., \& Faught, B. E. (2011). Physical activity and fitness in children with developmental coordination disorder: A systematic review. Research in Developmental Disabilities, 32(3), 894-910. https://doi.org/10.1016/j.ridd.2011.01.017

Rodriguez, M. C., Wade, T. J., Veldhuizen, S., Missiuna, C., Timmons, B., \& Cairney, J. (2019). Emotional and behavioral problems in 4- and 5-year old children with and without motor delays. Frontiers in Pediatrics, 7, 474. https://doi.org/10.3389/fped.2019.00474

Stanford, T. (2020). deltacomp: Functions to analyse compositional data and produce confidence intervals for relative increases and decreases in the compositional components (R package version 0.2.1.) [Computer software].

Tremblay, M. S. (2020). Introducing 24-hour Movement Guidelines for the Early Years: A new paradigm gaining momentum. Journal of Physical Activity and Health, 17(1), 92-95. https://doi.org/10.1123/jpah.2019-0401 
Tremblay, M. S., Chaput, J.-P., Adamo, K. B., Aubert, S., Barnes, J. D., Choquette, L., Duggan, M., Faulkner, G., Goldfield, G. S., Gray, C. E., Gruber, R., Janson, K., Janssen, I., Janssen, X., Jaramillo Garcia, A., Kuzik, N., LeBlanc, C., MacLean, J., Okely, A. D., ... Carson, V. (2017). Canadian 24-Hour Movement Guidelines for the Early Years (04 years): An integration of physical activity, sedentary behaviour, and sleep. BMC Public Health, 17(5), 874. https://doi.org/10.1186/s12889-017-4859-6

Trost, S. G., Pate, R. R., Freedson, P. S., Sallis, J. F., \& Taylor, W. C. (2000). Using objective physical activity measures with youth: How many days of monitoring are needed? Medicine and Science in Sports and Exercise, 32(2), 426-431. https://doi.org/10.1097/00005768-200002000-00025

van Buuren, S., \& Groothuis-Oudshoorn, K. (2011). mice: Multivariate imputation by chained equations in R. Journal of Statistical Software, 45(1), 1-67. https://doi.org/10.18637/jss.v045.i03 van den Boogaart, K. G., \& Tolosana-Delgado, R. (2008). "compositions": A unified R package to analyze compositional data. Computers \& Geosciences, 34, 320-338. https://doi.org/10.1016/j.cageo.2006.11.017 
MOVEMENT COMPOSITIONS AND MENTAL HEALTH

Table 1. Descriptive statistics for demographic, behavioural and mental health variables.

\begin{tabular}{|c|c|c|c|}
\hline & $\begin{array}{l}\text { Total Sample } \\
\qquad(N=589)\end{array}$ & $\begin{array}{c}\mathrm{TD} \\
(n=301)\end{array}$ & $\begin{array}{c}\text { rDCD } \\
(n=288)\end{array}$ \\
\hline Age & $4.94(0.59)$ & $5.00(0.61)$ & $4.87(0.56)$ \\
\hline Sex (male) & $338(57.4)$ & 145 (48.2) & $193(67.0)$ \\
\hline BMI z-score & $56.07(27.14)$ & $54.29(27.23)$ & $57.93(26.96)$ \\
\hline \multicolumn{4}{|l|}{ Household income } \\
\hline$<\$ 50,000$ & $78(13.2)$ & 35 (11.6) & $43(14.9)$ \\
\hline$\$ 50,000-\$ 99,999$ & $178(30.2)$ & $90(29.9)$ & $88(30.6)$ \\
\hline$\$ 100,00-\$ 150,000$ & $184(31.2)$ & $88(29.2)$ & $96(33.3)$ \\
\hline$\geq \$ 150,000$ & $149(25.3)$ & $88(29.2)$ & $61(21.2)$ \\
\hline \multicolumn{4}{|l|}{ Race/Ethnicity } \\
\hline White & $477(81.0)$ & $238(79.1)$ & $239(83.0)$ \\
\hline Asian & $36(6.1)$ & $17(5.6)$ & $19(6.6)$ \\
\hline Middle Eastern & $7(1.2)$ & $4(1.3)$ & $3(1.0)$ \\
\hline Black & $12(2.0)$ & $11(3.7)$ & $1(0.3)$ \\
\hline Indigenous & $3(0.5)$ & $3(1.0)$ & $0(0.0)$ \\
\hline Latino & $5(0.8)$ & $1(0.3)$ & $4(1.4)$ \\
\hline Mixed/Other & $49(8.3)$ & $27(9.0)$ & $22(7.6)$ \\
\hline MABC-2 Percentile & $33.17(29.12)$ & $56.18(23.40)$ & $9.13(5.48)$ \\
\hline \multicolumn{4}{|c|}{ Absolute accelerometer measured time-use (mins/day) } \\
\hline Sedentary Time & $451.24(45.55)$ & $451.69(44.30)$ & $450.78(46.90)$ \\
\hline LPA & $200.90(27.83)$ & $199.75(28.38)$ & $202.10(27.25)$ \\
\hline
\end{tabular}


MOVEMENT COMPOSITIONS AND MENTAL HEALTH

$\begin{array}{llll}\text { MVPA } & 71.81(19.66) & 71.97(20.85) & 71.63(18.38)\end{array}$

$\begin{array}{lll}\text { Total Wear Time } & 723.92(41.72) & 723.34(40.95)\end{array}$

Mean time-use composition (\% of wear time)

$\begin{array}{llll}\text { Sedentary Behaviour } & 62.33(5.16) & 62.47(5.30) & 62.19(5.02)\end{array}$

$\begin{array}{lll}\text { LPA } & 27.75(3.50) \quad 27.61(3.54) \quad 27.90(3.46)\end{array}$

$\begin{array}{llll}\text { MVPA } & 9.92(2.65) & 9.94(2.78) & 9.90(2.52)\end{array}$

$\begin{array}{lll}\text { Internalizing Problems } \quad 48.02(9.82) & 46.52(9.08) \quad 49.60(10.33)\end{array}$

Externalizing Problems $\quad 45.55(9.27) \quad 43.58(8.44) \quad 47.61(9.66)$

LPA $=$ light physical activity. MVPA = moderate-to-vigorous physical activity. Values in table represent means with standard deviations in parentheses or total cases with percent in parentheses. 
MOVEMENT COMPOSITIONS AND MENTAL HEALTH

Table 2. Associations between movement compositions and indicators of mental health

\begin{tabular}{|c|c|c|c|c|}
\hline & \multicolumn{4}{|c|}{ Indicators of Mental Health } \\
\hline & \multicolumn{2}{|c|}{ Internalizing Problems } & \multicolumn{2}{|c|}{ Externalizing Problems } \\
\hline & $B(\mathrm{SE})$ & $p$ value & $B(\mathrm{SE})$ & $p$ value \\
\hline Sedentary & $-0.26(2.59)$ & 0.92 & $-5.24(2.44)$ & 0.03 \\
\hline \multicolumn{5}{|l|}{ behaviour } \\
\hline Light physical & $3.08(3.68)$ & 0.40 & $4.16(3.64)$ & 0.25 \\
\hline \multicolumn{5}{|l|}{ activity } \\
\hline Moderate-to- & $-2.82(2.10)$ & 0.18 & $1.08(2.15)$ & 0.62 \\
\hline \multicolumn{5}{|l|}{ vigorous } \\
\hline physical activity & & & & \\
\hline
\end{tabular}

Significant associations are bolded. 

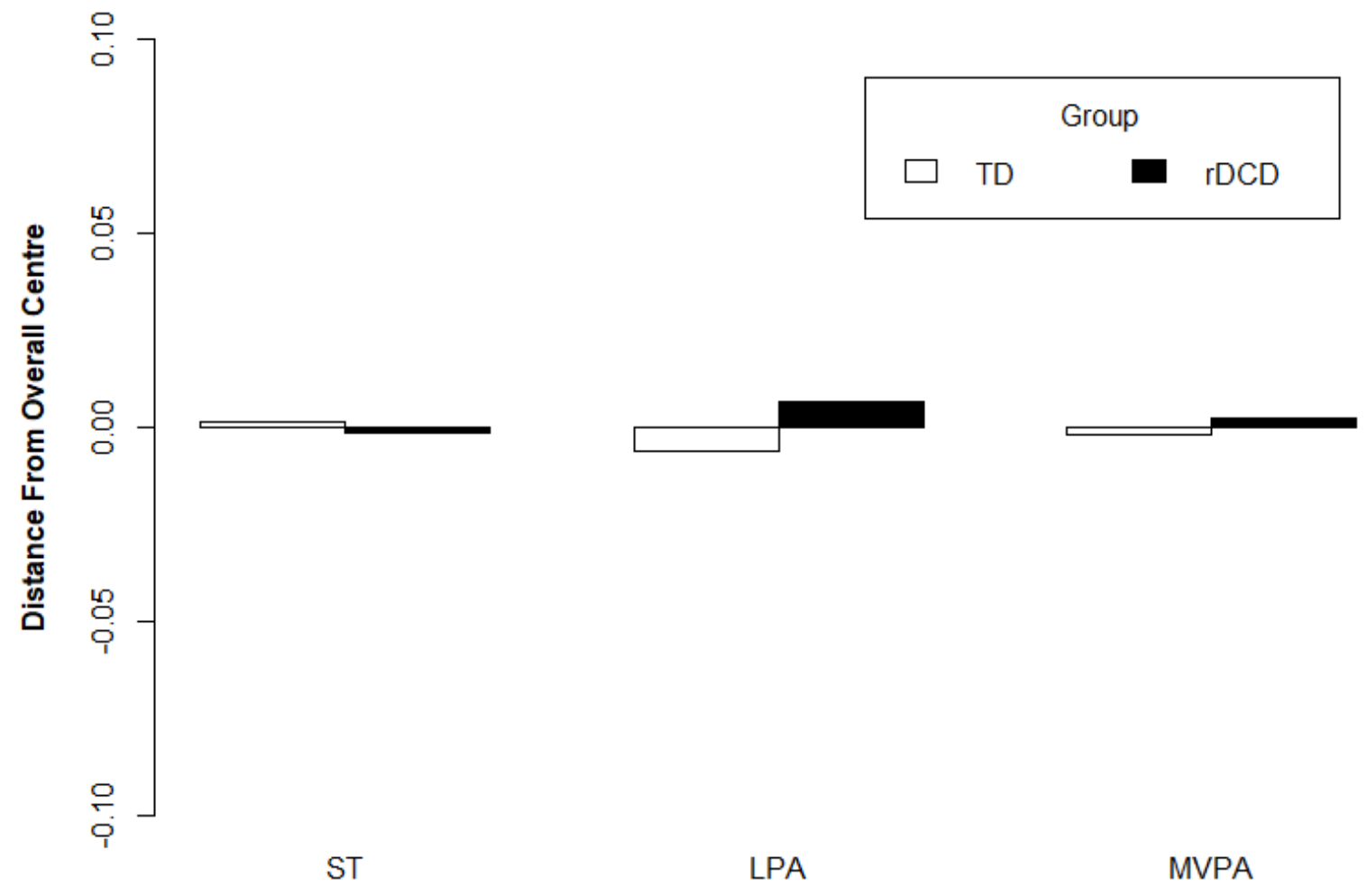

Movement Behaviours

Figure 1. Compositional analysis of the mean time spent in sedentary time (ST), light physical activity (LPA) and moderate-to-vigorous physical activity (MVPA) with respect to the overall mean time composition, by group. 
A) Movement Composition of TD Children

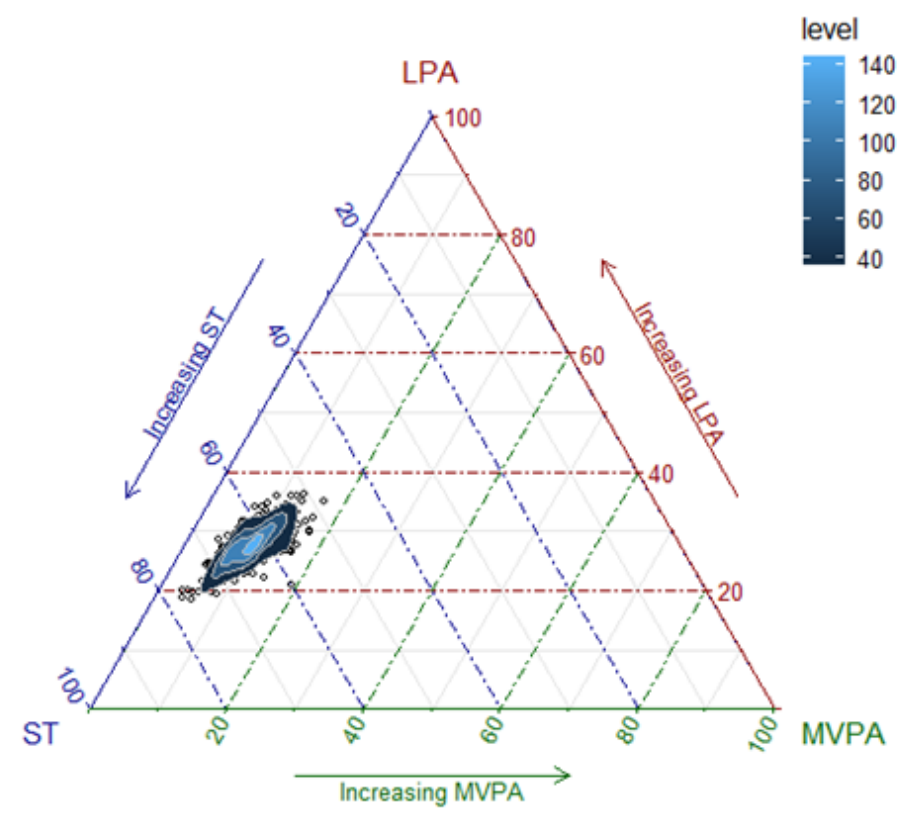

B) Movement Composition of rDCD Children

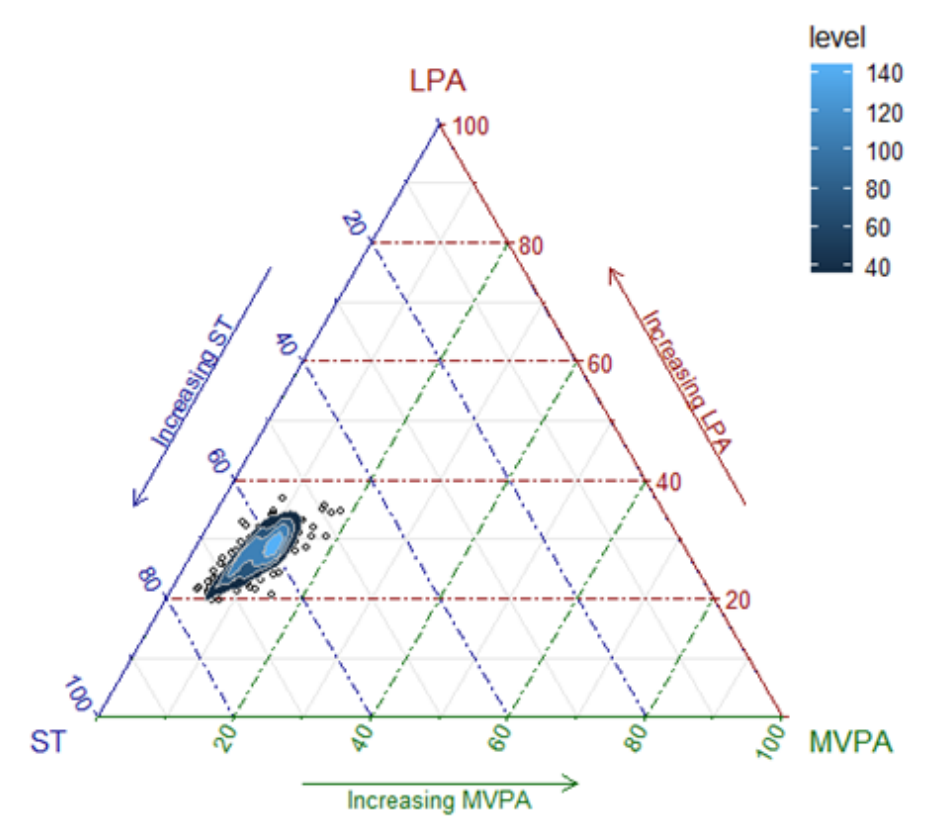

Figure 2. Ternary plots of the sample compositions of time spent in sedentary time (ST), light physical activity (LPA) and moderateto-vigorous physical activity (MVPA), for TD (A) and rDCD children (B) with 2D kernel density estimations. 
A)

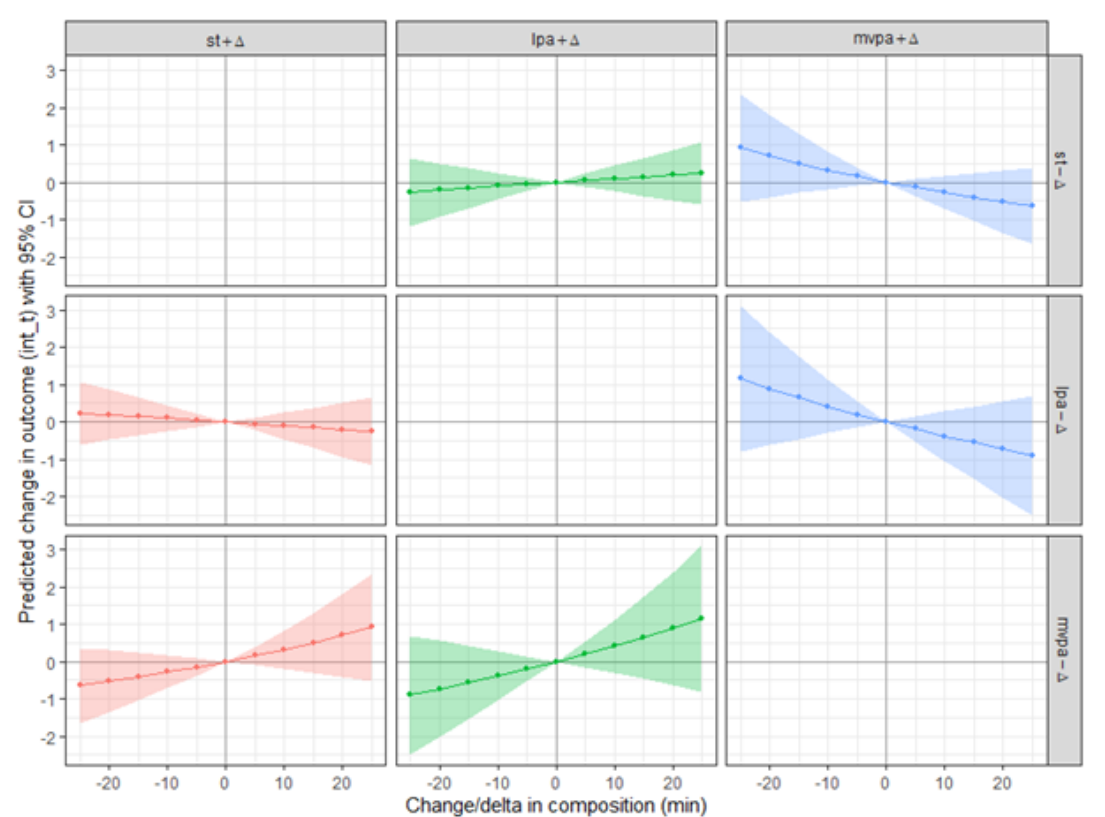

B)

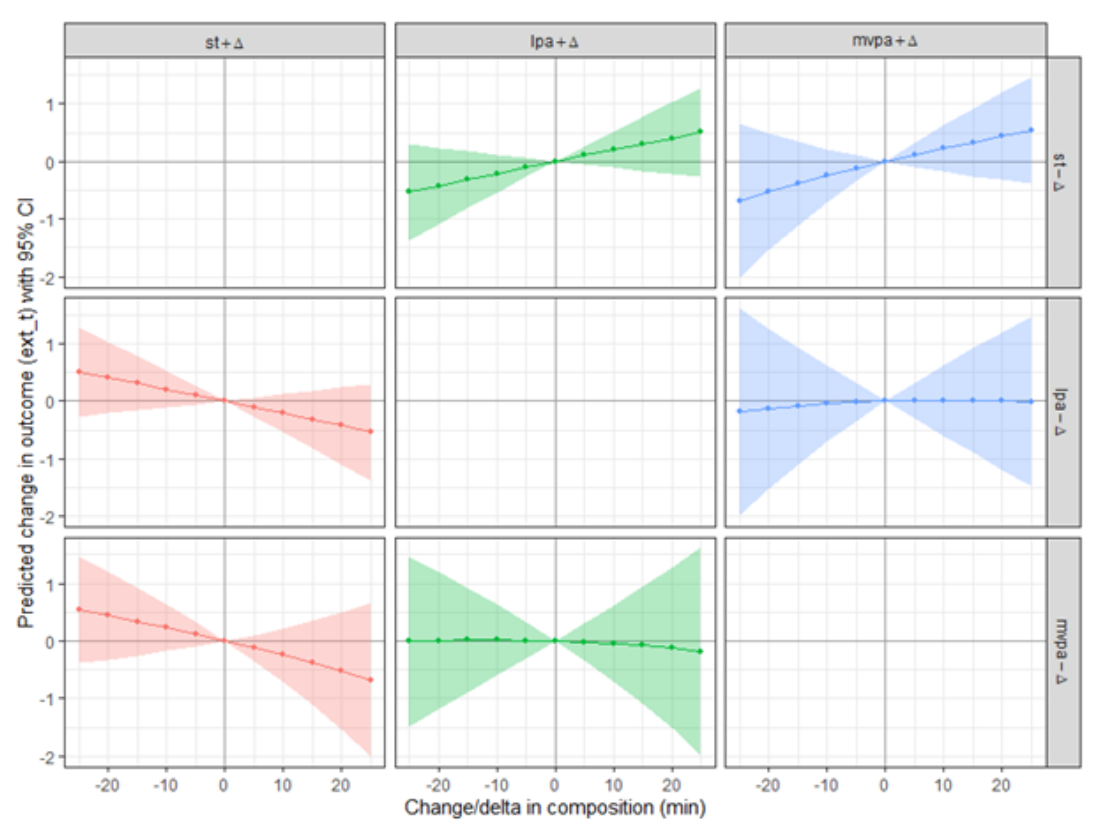

Figure 3. The impact of reallocating time ( 5 to $25 \mathrm{~min}$ ) between movement behaviours on internalizing problems (A) and externalizing problems (B). 